\title{
Controllable valley filter in graphene topological line defect with magnetic field
}

Hongyu Tian ( $\nabla$ tianhy2010@163.com )

Linyi university

Chongdan Ren

Zunyi Normal College

Benhu Zhou

Shaoyang University

Weitao Lu

Linyi University

Yunfang Li

Linyi University

Daoyong Li

Linyi University

Sake Wang

Jinling Institute of Technology

Lin Zhang

Nanjing Forestry University

\section{Nano Express}

Keywords: line defect, graphene, valley polarization

Posted Date: February 11th, 2020

DOI: https://doi.org/10.21203/rs.2.23169/v1

License: (c) (i) This work is licensed under a Creative Commons Attribution 4.0 International License.

Read Full License 


\title{
Controllable valley filter in graphene topological line defect with magnetic field
}

\author{
ChongDan Ren ${ }^{1}$, Lin Zhang ${ }^{2}$, Benhu Zhou ${ }^{3}$, Weitao $\mathrm{Lu}^{4}$, Yunfang $\mathrm{Li}^{5}$, Daoyong $\mathrm{Li}^{4}$, Sake Wang ${ }^{6,7}$ and \\ Hongyu $\operatorname{Tian}^{4 * \dagger}$
}

\author{
${ }^{*}$ Correspondence: \\ tianhongyu@lyu.edu.cn, \\ lidaoyong@lyu.edu.cn \\ ${ }^{4}$ School of Physics and Electronic \\ Engineering, Linyi University, Linyi \\ 276005, China \\ Full list of author information is \\ available at the end of the article \\ ${ }^{\dagger}$ Equal contributor
}

\begin{abstract}
First part title: The extended line defect of graphene is an extraordinary candicate in valleytronics while the high valley polarization can only occur for electrons with high incidence angles which brings about tremendous challenges to experimental relization. In this paper, we propose a simple scheme to filter one conical valley state by applying a local magnetic field. It is found that due to the movement of the Dirac points, the transmission profiles of the two valleys are shifted along the injection-angle axis at the same pace, resulting in the peak transmission of one valley state being reduced drastically while remaining unaffected for the other valley state, which induces nearly perfect valley polarization. This scheme can be easily implemented in experiments and plays a key role in graphene valleytronics.
\end{abstract}

Keywords: line defect; graphene; valley polarization

\section{Introduction}

Graphene, a single atomic layer of graphite, exhibits peculiar electronic structure[1, $2]$. The valence and conduction bands of graphene touch each other at $K$ and $K^{\prime}$ points, the two inequivalent corners of the hexagonal Brillouin zone. The low-energy electron around the two points can be described by the massless Dirac equation. The two Dirac points, called $K$ and $K^{\prime}$ valleys, were suggested as an information carrier and played an important role in the recently emerged valleytronics[3].

The polycrystalline which is composed of different single-crystal grains separated by grain boundaries $[4,5]$, always appear in graphene samples synthesized at large scale. The polycrystalline graphene is the nature valley filter and has received considerable attention in recent years $[6,7,4]$. V. Hung Nguyen found that the perfect valley polarization can emerge in a wide range of transmission directions in the presence of strain[6]. Obviously, this is the inevitable consequence when the two Dirac points move in opposite directions in the momentum space under strain. However, an interesting question arises naturally: what will happen when the two Dirac points move in the same direction in the momentum space.

One particular type of polycrystalline graphene, as learned from electrons, is the extended line defect containing carbon atom pentagons and octagons. Many peculiar electronic characteristics have been explored theoretically due to the unique structure of this defect $[8,9,10,11,12,13]$ Furthermore, such extended linear defect has been experimentally observed on a graphene layer grown on a metallic 
substrate[14]. The line defect has important applications in valleytronics. D. Gunlycke and C. T. White pointed out that a valley polarization of nearly $100 \%$ can be achieved by scattering off a line defect[7], attracting intense interest in the physics community. However, perfect valley polarization can only appear for the electrons with large incident angles, and the efficiency is reduced or even completely disappears when an electron is transmitted through the line defect perpendicularly. This effect indicates that the electron must always follow the direction of the line defect to maintain a high valley polarization, bringing about major challenges for the experimental studies of this phenomenon. Not confined to the line defect, the dilemma also exists in other polycrystalline graphene[6] and this is also why the strain should be taken into account.

As an effective means of field regulation and control of valley polarization, the function of the magnetic field has been widely explored. For instance, a quantizing magnetic field generating the valley-polarized quantum Hall state in the graphene $p-n$ junction has been investigated in theoretical studies $[15,16,17,18,19]$ and confirmed in experiment with a high magnetic field[20]. Of course, the valley-polarized Landau level can be created under an even stronger magneitc field[21]. In addition, the magnetic field can also be used to realize the valley polarization by introducing the vector potential to break the symmetry of the two valleys[22, 23] or coupling with the orbital magnetic moment in a system with broken inversion symmetry to generate the valley-dependent Zeeman interaction[24]. However, a surprising function related to the magnetic field is rarely mentioned: the transmission profiles are deflected to one side along the incidence angle axis due to movement of the Dirac points in the magnetic field, and the peak transmission is shifted to the left or right of the normal axis depending on the direction of the magnetic field[25, 26, 27]. It is expected that this phenomenon will significantly influence the transmission characteristics of the valley states in the line defect because the transmission coefficient is dependent of the incidence angle, the peak transmission of one valley is situated at one end of the transmission image while situated at the opposite end for the other valley, and the transmission enhances(weakens) from 0(1) to 1(0) as the angle varies through the transmission image for the two valleys[7, 15]. Currently, the research focus of experimental[28] and theoretical[29, 30, 12] attention in this field are mainly on the quantum Hall effect(QHE) $[28,29,30]$ and the QH boundary states[12] under a perpendicular magnetic field, whereas the manipulation of the valley state with the magnetic field is still in its infancy.

In this paper, we propose a simple technique to filtrate one valley state in the line defect of graphene with a magnetic field. Interestingly, it is found that, in the presence of a perpendicular magnetic field, the transmission curves of the two valleys shift rightward together with the same pace, resulting in the peak transmission of the $K$ valley with unit transmission shifts along the incidence angle axis, while that of the $K^{\prime}$ valley diminishes rapidly. Therefore, the transmission of the $K^{\prime}$ valley is enormously restrained, and nearly $100 \%$ valley polarization can be realized. The physical mechanism of this phenomenon is that due to the movement of the two Dirac points in the momentum space, the eject angle of the $K$ valley state through the scattering region can always maintain the preferred angle $(-\pi / 2)$, whereas the outgoing angle of the $K^{\prime}$ valley is far from the preferred angle $(\pi / 2)$ due to the 
momentum conservation. The scheme does not require a high magnetic field and can be easily implemented in the experiment.

\section{Model}

We model the electronic structure of the graphene line defect shown in Fig. 1 by a nearest-neighbor tight-binding Hamiltonian[13, 31, 32]

$$
H=\sum_{i}\left(\epsilon_{i}+w_{i}\right) c_{i}^{\dagger} c_{i}+t \sum_{\langle i, j\rangle} c_{i}^{\dagger} c_{j}+\tau_{2} \sum_{\langle\gamma \delta\rangle} c_{i_{y}, \gamma}^{\dagger} c_{i_{y}, \delta}+\tau_{1} \sum_{\langle i, \gamma\rangle} c_{i}^{\dagger} c_{i_{y}, \gamma}+H . c .,(1)
$$

where $c_{i}^{\dagger}$ and $c_{i_{y}, \gamma / \delta}^{\dagger}$ represent the electron creation operator at site $i$ and the line defect, respectively. Here, $\epsilon_{i}$ in the first term of Eq. (1) is the on-site energy (i.e.,the energy of the Dirac point), which can be controlled experimentally by the gate voltage, and $w_{i}$ is the random disorder potential uniformly distributed in the interval $w_{i} \in[-W / 2, W / 2] t$, with $W$ being the disorder strength. The second term in Eq. (1) represents the nearest-neighbor interaction in pristine graphene, the third term denotes the interaction between the two atoms in the line defect and the fourth one is the hopping term between the defect atom and its nearest neighbor in pristine graphene. $t, \tau_{1}$ and $\tau_{2}$ represent different nearest-neighbor hopping energies, as shown in Fig. 1(c), and $\langle\cdots\rangle$ runs over all the nearest-neighbor hopping sites. This type of defect conserves the coordination number, which implies variations less than $5 \%$ in $t$, suggesting that $t$ values could be considered nearly identical and it is reasonable to set $\tau_{1} \approx \tau_{2} \approx t$ (for which $t \approx 3.1 \mathrm{eV}$ )[13,33]. In the presence of a perpendicular magnetic field $\vec{B}$, a Peierls phase factor $\phi_{i j}=2 \pi \int_{i}^{j} \vec{A} \cdot d \vec{l} / \phi_{0}$ should be added in the hopping interactions with the vector potential $\left(A_{x}, A_{y}\right)=(0, B x)$, where $\phi_{0}=\hbar / e$, and the magnetic length $l_{0}=\sqrt{\hbar / e B} \approx 26 \mathrm{~nm}$ for $B=1 \mathrm{~T}$ [34]. It is supposed that the magnetic field is applied adjacent to the line defect, as shown in Fig. 1(a).

The line defect of graphene is shown in Fig. 1(a), which extends immensely along the $y$ direction. The translational symmetry of the lattice structure along the $y$ direction indicates that $k_{y}$ is a conserved quantity and that the creation (annihilation) operators can be rewritten as follows, according to the Fourier transformation:

$$
\begin{aligned}
c_{i}^{\dagger} & =\sum_{k_{y}} c_{k_{y}, i_{x}} e^{-2 i k_{y} i_{y} a}, c_{i}=\sum_{k_{y}} c_{k_{y}, i_{x}} e^{2 i k_{y} i_{y} a} \\
c_{i_{y}, \gamma}^{\dagger}=\sum_{k_{y}, \gamma} c_{k_{y}, \gamma}^{\dagger} e^{-2 i k_{y} i_{y} a}, c_{i_{y}, \gamma} & =\sum_{k_{y}, \gamma} c_{k_{y}, \gamma} e^{2 i k_{y} i_{y} a} .
\end{aligned}
$$

Then, the Hamiltonian matrix in Eq. (1) is decoupled into $H=\sum_{k_{y}} H_{k_{y}}$, where $H_{k_{y}}$ can be described in the following form:

$$
\begin{array}{r}
H_{k_{y}}=-\sum_{i} \varphi_{i, 1}^{\dagger} \hat{T}_{1} \varphi_{i, 2}-\sum_{i} \varphi_{i, 2}^{\dagger} \hat{T}_{2} \varphi_{i, 3} \\
-\sum_{i} \varphi_{i, 3}^{\dagger} \hat{T}_{1}^{\dagger} \varphi_{i, 4}-\sum_{i \neq-1} \varphi_{i, 4}^{\dagger} \hat{T}_{2}^{\dagger} \varphi_{i+\hat{x}, 1} \\
-\varphi_{\overline{1}, 4}^{\dagger} \hat{T}_{2} \varphi_{0}-\varphi_{0}^{\dagger} \hat{T}_{2} \varphi_{1,1}-\varphi_{0}^{\dagger} \hat{T}_{3} \varphi_{0}+\text { h.c. }+\sum_{i}\left(\epsilon_{i}+w_{i}\right) \varphi_{i}^{\dagger} \varphi_{i},
\end{array}
$$




$$
\hat{T}_{1}=\left(\begin{array}{cc}
1 & 1 \\
e^{-i 2 \theta} & 1
\end{array}\right), \hat{T}_{2}=\left(\begin{array}{ll}
1 & 0 \\
0 & 1
\end{array}\right), \hat{T}_{3}=\left(\begin{array}{ll}
0 & 1 \\
1 & 0
\end{array}\right)
$$

where $\varphi_{i, \gamma}^{\dagger}=\left[c_{k_{y}, i, \gamma, 1}^{\dagger}, c_{k_{y}, i, \gamma, 2}^{\dagger}\right](\bar{i}=-i)$, and $\hat{x}$ represents the unit length between the neighboring supercells at the graphene part. Here, $i$ represents the position of a supercell, $\gamma$ takes the integer number from 1 to 4 denoting the different columns in a supercell, and $1 / 2$ in $c_{k_{y}, i, \gamma, 1 / 2}^{\dagger}$ corresponds to the up/down site in the same column in Fig. 1(c).

We also explore the non-equilibrium Green's function technique to calculate the transmission coefficient[32],

$$
T_{k_{y}}(E)=\operatorname{Tr}\left[\left(\Gamma_{L}\right)\left(G^{r}\right)\left(\Gamma_{R}\right)\left(G^{a}\right)\right]
$$

where $G^{r}\left(G^{a}\right)$ is the retarded(advanced) Green's function related to the line-defect Hamiltonian and $\Gamma_{L / R}=i\left(\Sigma_{L / R}^{r}-\Sigma_{L / R}^{a}\right)$ with the retarded/advanced self-energy $\Sigma_{L / R}^{r} / \Sigma_{L / R}^{a}$ of the left(right) lead. Note that here the left/right lead is represented by a semi-infinite quasi-one dimensional graphene lattice sketched in Fig. 1(c), and the scattering region includes the line defect and the $B$ region with width $L$, as shown in Fig. 1(a). The two Dirac points, $K$ and $K^{\prime}$, are located at $[0, \pm \pi / 3 a]$ for graphene with a line defect. Therefore, the momentum can be expanded at $k_{x}=q_{x}$ and $k_{y}=q_{y} \pm \pi / 3 a$ where $q_{x}\left(q_{y}\right)$ represents the group velocity of electrons along the $x(y)$ direction. Combining this with the linear dispersion relation of the Dirac electrons $E=\frac{\sqrt{3} q}{2}=\frac{\sqrt{3} \sqrt{q_{x}^{2}+q_{y}^{2}}}{2}$, the transmission coefficients can be calculated as a function of the electron incident angle $\alpha$ with $\alpha=\arctan \left(q_{y} / q_{x}\right)$.

We would now like to briefly illustrate the shift of the peak transmission of the two valleys under the $B$-field, as shown in Fig. 1(b). In the presence of a perpendicular $B$-field, the two Dirac points are shifted along the $k_{y}$-axis by an amount of $\Delta k=$ $e B L / \hbar[25,26]$. It is well known that the transmission of the $K\left(K^{\prime}\right)$ valley adopts the peak value $T_{K}\left(T_{K^{\prime}}\right) \approx 1$ as the scattering angle is about $-\pi / 2(\pi / 2)$, whereas it will decrease when the scattering angle is away from $-\pi / 2(\pi / 2)$. It is clear that when an electron from the $K^{\prime}$ valley injects with an incidence angle $\pi / 2$, the actual outgoing angle $\alpha_{1}$ through the $B$-field will be less than $\pi / 2$ according to the conservation of $k_{y}$, as shown in Fig. 1(a) and the lower panel of Fig. 1(b). Hence, the transmission of $K^{\prime}$ valley through the scattering region will be greatly restrained which can be depicted visually by the line width variation of the blue arrow shown in Fig. 1(b). However, the situation is completely different for the $K$ valley. It is shown in the upper panel of Fig. 1(b) that regardless of the magnitude of $\Delta k$, there always exists such an incident angle that makes the outgoing angle through the $B$-field equal to $-\pi / 2$ due to the conservation of $k_{y}$. Therefore, the peak transmission of the $K$ valley $T_{K}=1$ can always survive as $\Delta k$ increases, as depicted by the red arrow with no variation in line width. According to the conservation of $k_{y}$, $q \sin \alpha=q \sin (-\pi / 2)+\Delta k$, one can obtain the relation between $\alpha$ and $\Delta k$ for the peak transmission $T_{K}=1$ :

$$
\alpha=\arcsin \left(\frac{\Delta k}{q}-1\right)
$$




\section{Numerical Results}

In the following, we will numerically investigate the shift of the transmission curves and the valley polarization phenomenon in the line defect of graphene under a perpendicular magnetic field. In the calculations of the transmission coefficients, we set $\tau_{2}=\tau_{1}=t=1$ as the energy unit, the Fermi energy $E_{f}=0.01 t$, the width of the $B$-field $L=174$ and the disorder average is carried out over 4000 sample configurations.

In Fig. 2, the transmission coefficient is mapped as a function of the electron's incident angle $\alpha$ for different magnetic fields. As $B=0$ (the blue line), the transmission coefficient of $K / K^{\prime}$ valley $T_{K} / T_{K^{\prime}}$ varies slowly from $1 / 0$ to $0 / 1$ with $\alpha$, and the peak transmission $T_{K} / T_{K^{\prime}} \approx 1$ appears at $\alpha \approx-\frac{\pi}{2} / \frac{\pi}{2}$, as shown in Fig. $2(\mathrm{a}) /(\mathrm{b})$. Clearly, the valley polarization can only appear for high incident angles. When a $B$-field is applied, the transmission profiles combined with the peak transmission of the two valleys are shifted along the incidence angle axis from negative angles to positive angles with the same pace, and the deviation continues as the $B$-field strengthens, as depicted by the curves with different colors in Fig. 2. Consequently, the peak transmission of the $K$ valley $T_{K}=1$ is maintained well, while that of the $K^{\prime}$ valley weakens and even disappears as $B$ increases. For instance, the maximum transmission coefficient of the $K$ valley with $T_{K}=1$ appears at $\alpha=-0.04 \pi$ when $B=1 \mathrm{~T}$, while this position moves to $\alpha=0.25 \pi$ as $B=2.1 \mathrm{~T}$ and even up to $\alpha=0.5 \pi$ as $B=2.31 \mathrm{~T}$. This is because as an electron injects with an angle $\alpha=-0.04 \pi(0.5 \pi)$, it will eject from the scattering region with an angle $-\pi / 2$ at $B=1 \mathrm{~T}(2.31 \mathrm{~T})$. As $B$ further strengthens, the peak transmission of the $K$ valley state still has a considerable magnitude which can reach 0.96 as $B=2.35 \mathrm{~T}$, as shown in Fig. 2(a). However, the peak transmission of the $K^{\prime}$ valley decreases rapidly with increasing $B$, and the maximum value found at $B=2.1 \mathrm{~T}$ is less than 0.04 , which indicates a nearly $100 \%$ valley polarization. Therefore, the nearly $100 \%$ valley polarization can always appear in a certain range of the magnetic field $(2.1 \mathrm{~T} \sim 2.35 \mathrm{~T})$. This conclusion is consistent with the analytical results presented above.

To further interpret the shift of the transmission curve and the peak transmission in a $B$-field, we plot the transmission coefficients of the two valleys versus the incident angle $\alpha$ and $B$-field, as shown in Fig. 3. The relation between $\alpha$ and $B$ (or $\Delta k$ ) according to Eq. 6 as $T_{K}=1$ is also plotted, as depicted by the blue line in Fig. $3(\mathrm{a})$. It is shown that as $B$ strengthens, the critical incident angle $\alpha$ corresponding to $T_{K}=1$ gradually varies along the incident angle axis, which has the same variation tendency with the line obtained from Eq. 6 . However, as $\alpha>0.2 \pi$ it requires a higher magnetic field to realize the peak transmission $T_{K}=1$ compared with the theoretical analysis. This clearly indicates that the shift of the peak transmission and the valley polarization should be induced by the movement of the Dirac points in the $B$-field.

As mentioned above, nearly $100 \%$ valley polarization can always occur when the peak transmission of the $K$ valley $T_{K}=1$ appears as $\alpha=\pi / 2$. Since the nearly $100 \%$ valley polarization is related to the movement of the Dirac points which is dependent of the magnetic field $B$ and the width of the scattering region $L$, the valley polarization should occur in a certain range of $B$ and $L$. In Fig. 4 , we present 
the critical magnetic field $B_{c}$ and the corresponding width $L$ to realize $T_{K}=1$ as $\alpha=\pi / 2$ (the blue line) for different energies. It is found that $B_{c}$ decreases linearly as $L$ broadens while this phenomenon can only emerge in a certain range of $B$ and $L$. For instance, it appears in the in the ranges of $[2.26 \mathrm{~T}, 2.34 \mathrm{~T}]$ and $[164,184]$ at $E=0.01 t$ while in the region of $[0.55 \mathrm{~T}, 0.56 \mathrm{~T}]$ and $[334,376]$ for $E=0.005 t$, as shown in Fig. 4 . In fact, the nearly $100 \%$ valley polarization can always occur when the magnetic field $B$ is in such special range not confined to $B_{c}$. In the insert of Fig. 4 (b), we select a position randomly in these ranges(the red circle between the two dashed lines) and plot the the transmission curves of the two valleys. It is found that the peak transmission of the $K$ valley remains at 1 while it is less than 0.04 for the $K^{\prime}$ valley which indicates a nearly $100 \%$ valley polarization.

To conveniently detect the valley polarized current in the experiment, we present the ranges of $B$ and $L$ to realize the nearly $100 \%$ valley polarization with $T_{K}=1$ as $\alpha$ approaches $\pi / 2$ at different energies, as shown in Table 1 . It is found that at a lower energy, a weak magnetic field is required to generate the valley polarized current. In the experiment, it is easy to apply a magnetic field up to approximately $3 \mathrm{~T}$, and the scheme can be easily implemented in a real experiment for at least $E \leq 0.01 t$. During an experiment, one can analyze the valley-polarized electrical currents from the left to right lead with an experimentally measurable quantity such as the conductance, which can be given according to the Landauer-Büttiker formula $G_{K / K^{\prime}}=\frac{e^{2}}{h} \int_{-k_{F}}^{k_{F}} T_{K / K^{\prime}} \frac{d k_{y}}{2 \pi / L_{y}}=\frac{L_{y} e^{2} E}{2 h \pi \hbar v_{F}} \int_{-\pi / 2}^{\pi / 2} T_{K / K^{\prime}} \cos \alpha d \alpha$, where $L_{y}=0.492 \mathrm{~nm}$ is the sample size along the $y$ direction, $v_{F} \approx 1.0 \times 10^{6} \mathrm{~m} / \mathrm{s}$ is the Fermi velocity, $\hbar=h / 2 \pi$ is the reduced Planck constant with $h=4.13566743 \times 10^{15} \mathrm{eV} \cdot \mathrm{s}$, and $E$ is the on-site energy of the incident electrons. For a homogeneous system where the electrons in the whole device have the same on-site energy, the conductance may be weak, approximately $G_{K} \approx 5.0 \times 10^{-3} e^{2} / h$ at $E=0.01 t$, which seems difficult to detect. However, as the electrons' on-site energy in the left electrode $E_{L}$ is raised, the valley polarization effect is essentially unaffected due to the conservation of $k_{y}$ while the conductance can be increased dozens of times, which can be detectable in a real experiment. For instance, as the on-site energy in the left electrode is raised to $E_{L}=0.2 t$ (or even higher), the maximal value of $T_{K}$ can still remain at a large magnitude(about 0.9), while that of $T_{K^{\prime}}$ is still less than 0.05, as shown in Fig. 5, which manifests a perfect valley polarization. However, $\int_{-\pi / 2}^{\pi / 2} T_{K} \cos \alpha d \alpha$ remains almost unchanged compared with the $E_{L}=0.01 t$ curve, and the magnitude of $G_{K}$ can be up to the order of $0.1 e^{2} / h$, which can be detectable in practical experiments.

In a real device, the disorder is always present due to the existence of adatoms or vacancies. To simulate a more realistic line defect, a random potential with a uniform distribution in the range $[-W / 2, W / 2] t$, with $W$ being the disorder strength, should be introduced on the scattering region to mimic a generic short-range disorder. In Fig. 6, we calculate the transmission coefficients of the two valleys for different disorder strengths. For weak disorder $(W<0.25), T_{K}$ changes only slightly compared with the clean sample. As $W$ increases, the maximal value of $T_{K}$ decreases, while it still has a considerable magnitude at strong disorder $\operatorname{strength}(W=0.65)$, as shown in Fig. 6(a). The disorder does not have a substantial influence on the transmission of the $K^{\prime}$ valley, as shown in Fig. 6(b). Thus, the pure valley current can be detected in a real experiment. 


\section{Conclusion}

In summary, we propose a new approach for achieving valley polarization in a graphene line defect by application of a magnetic field. In contrast to the previous techniques to achieve the valley polarization with a magnetic field, we consider a special transmission characteristic of graphene whereby the transmission curves shift along the incident angle axis in a magnetic field. The valley polarization can reach nearly $100 \%$ in a certain range of magnetic field. Superior to other schemes, it does not require a high magnetic field to create quantum Hall states, and the manufacturing technology is relatively simple and feasible. Moreover, theoretical work suggests that such an extended line defect can be produced in suspended graphene in a controlled manner and a predetermined location even without a catalyzing metal substrate[35]. We hope that our finding may provide a route for designing valleytronic devices with graphene.

Competing interests

The authors declare that they have no competing interests.

Author's contributions

HYT and CDR together carried out the physical idea and were major contributors in writing the manuscript. BHZ derived the algorithm to calculate the transmission coefficients with RSOC, HYT, and CDR carried out the numerical results of calculations. LZ and SKW provided guidance in improving the quality of the manuscript. WTL, YFL, and DYL participated in the result analysis and manuscript preparation. All authors reviewed the manuscript. All authors read and approved the final manuscript.

\section{Acknowledgements}

This work was supported by the NNSF of China (Nos. 11864047, 11547127, 11704165 and 11974153), China Scholarship Council (No. 201908320001), the Science Foundation of Guizhou Provincial Education Department (No. QJHKYZ[2016]092), the Natural Science Foundation of Shandong Province (Grant Nos. ZR2017JL007, ZR201807060735), the Project ofShandong Province Higher Educational Science and Technology Program(No. J18KB102), the Major Research Project for Innovative Group of Education Department of Guizhou Province (no. KY[2018]028), the Scientific Research Fund of Hunan Provincial Education Department (Grant No. 17A193), and the Science Foundation of Jinling Institute of Technology (No. 40620064).

\section{Author details}

${ }^{1}$ Department of Physics, Zunyi Normal University, Kweichow 563002, China. ${ }^{2}$ College of Science, Nanjing Forestry University, Nanjing 210037, China. ${ }^{3}$ Department of Physics, Shaoyang University, Shaoyang 422001, China. ${ }^{4}$ School of Physics and Electronic Engineering, Linyi University, Linyi 276005, China. ${ }^{5}$ School of Mechanical \& Vehicle Engineering, Linyi University, Linyi 276005, China. ${ }^{6}$ College of Science, Jinling Institute of Technology, Nanjing 211169, China. ${ }^{7}$ Department of Physics, Tohoku University, Sendai 980-8578, Japan.

\section{References}

1. Novoselov, K.S., Geim, A.K., Morozov, S.V., Jiang, D., Zhang, Y., Dubonos, S.V., Grigorieva, I.V., Firsov, A.A.: Electric field effect in atomically thin carbon films. Science (New York, N.Y.) 306(5696), 666-669 (2004). doi:10.1126/science.1102896

2. Wallace, P.R.: The band theory of graphite. Physical Review 71(9), 622-634 (1947) doi:10.1103/PhysRev.71.622

3. Topsakal, M., Bagci, V.M.K., Ciraci, S.: Current-voltage (i-v) characteristics of armchair graphene nanoribbons under uniaxial strain. Physical Review B 81(20), 205437 (2010). doi:10.1103/PhysRevB.81.205437

4. Yazyev, O.V., Chen, Y.P.: Polycrystalline graphene and other two-dimensional materials. Nature nanotechnology 9(10), 755-767 (2014). doi:10.1038/nnano.2014.166

5. Cummings, A.W., Duong, D.L., van Nguyen, L., van Tuan, D., Kotakoski, J., Barrios Vargas, J.E., Lee, Y.H., Roche, S.: Charge transport in polycrystalline graphene: challenges and opportunities. Advanced materials (Deerfield Beach, Fla.) 26(30), 5079-5094 (2014). doi:10.1002/adma.201401389

6. Nguyen, V.H., Dechamps, S., Dollfus, P., Charlier, J.-C.: Valley filtering and electronic optics using polycrystalline graphene. Physical review letters 117(24), 247702 (2016). doi:10.1103/PhysRevLett.117.247702

7. Gunlycke, D., White, C.T.: Graphene valley filter using a line defect. Physical review letters 106(13), 136806 (2011). doi:10.1103/PhysRevLett.106.136806

8. Phillips, M., Mele, E.J.: Zero modes on zero-angle grain boundaries in graphene. Physical Review B 91(12), 30 (2015). doi:10.1103/PhysRevB.91.125404

9. Rodrigues, J.N.B., Peres, N.M.R., Lopes dos Santos, J.M.B.: Scattering by linear defects in graphene: a tight-binding approach. Journal of physics. Condensed matter : an Institute of Physics journal 25(7), 075303 (2013). doi:10.1088/0953-8984/25/7/075303

10. Lin, X., Ni, J.: Half-metallicity in graphene nanoribbons with topological line defects. Physical Review B 84(7), 075461 (2011). doi:10.1103/PhysRevB.84.075461 
11. Innatsenka, S., Zozoulenko, I.V.: Electron interaction, charging, and screening at grain boundaries in graphene. Physical Review B 88(8), 085436 (2013). doi:10.1103/PhysRevB.88.085436

12. Yao, H.-B., Lü, X.-L., Zheng, Y.-S.: Quantum hall boundary state around the line defect in graphene. Physical Review B 88(23), 235419 (2013). doi:10.1103/PhysRevB.88.235419

13. Liu, Y., Song, J., Li, Y., Liu, Y., Sun, Q.-f.: Controllable valley polarization using graphene multiple topological line defects. Physical Review B 87(19), 195445 (2013). doi:10.1103/PhysRevB.87.195445

14. Lahiri, J., Lin, Y., Bozkurt, P., Oleynik, I.I., Batzill, M.: An extended defect in graphene as a metallic wire. Nature nanotechnology 5(5), 326-329 (2010). doi:10.1038/nnano.2010.53

15. Tworzydło, J., Snyman, I., Akhmerov, A.R., Beenakker, C.W.J.: Valley-isospin dependence of the quantum hall effect in a graphene $p-n$ junction. Physical Review B 76(3), 035411 (2007). doi:10.1103/PhysRevB.76.035411

16. Trifunovic, L., Brouwer, P.W.: Valley isospin of interface states in a graphene pn junction in the quantum hall regime. Physical Review B 99(20), 205431 (2019). doi:10.1103/PhysRevB.99.205431

17. Sekera, T., Bruder, C., Mele, E.J., Tiwari, R.P.: Switchable valley filter based on a graphene $p-n$ junction in a magnetic field. Physical Review B 95(20), 205431 (2017). doi:10.1103/PhysRevB.95.205431

18. Peterson, M.R., Nayak, C.: Effects of landau level mixing on the fractional quantum hall effect in monolayer graphene. Physical review letters 113(8), 086401 (2014). doi:10.1103/PhysRevLett.113.086401

19. Fräßdorf, C., Trifunovic, L., Bogdanoff, N., Brouwer, P.W.: Graphene pn junction in a quantizing magnetic field: Conductance at intermediate disorder strength. Physical Review B 94(19), 424 (2016). doi:10.1103/PhysRevB.94.195439

20. Handschin, C., Makk, P., Rickhaus, P., Maurand, R., Watanabe, K., Taniguchi, T., Richter, K., Liu, M.-H., Schönenberger, C.: Giant valley-isospin conductance oscillations in ballistic graphene. Nano letters 17(9), 5389-5393 (2017). doi:10.1021/acs.nanolett.7b01964

21. Zhang, Y., Jiang, Z., Small, J.P., Purewal, M.S., Tan, Y.-W., Fazlollahi, M., Chudow, J.D., Jaszczak, J.A., Stormer, H.L., Kim, P.: Landau-level splitting in graphene in high magnetic fields. Physical review letters 96(13), 136806 (2006). doi:10.1103/PhysRevLett.96.136806

22. Lu, W.-T., Yang, Q., Yao, L.-F., Cui, D., Tian, H.-Y.: Giant magnetoresistance and spin-valley polarization of dirac fermions modulated by magnetic fields. Applied Physics Express 12(2), 025007 (2019). doi: $10.7567 / 1882-0786 /$ aaff 25

23. Smoleński, T., Goryca, M., Koperski, M., Faugeras, C., Kazimierczuk, T., Bogucki, A., Nogajewski, K., Kossacki, P., Potemski, M.: Tuning valley polarization in a wse2 monolayer with a tiny magnetic field. Physical Review X 6(2), 995 (2016). doi:10.1103/PhysRevX.6.021024

24. Park, C.: Magnetoelectrically controlled valley filter and valley valve in bilayer graphene. Physical Review Applied 11(4), 044033 (2019). doi:10.1103/PhysRevApplied.11.044033

25. Li, Y., Wan, Q., Peng, Y., Wang, G., Qian, Z., Zhou, G., Jalil, M.B.A.: The effect of magnetic field on chiral transmission in p-n-p graphene junctions. Scientific reports 5, 18458 (2015). doi:10.1038/srep18458

26. Bala Kumar, S., Jalil, M.B.A., Tan, S.G.: Klein tunneling in graphene systems under the influence of magnetic field. Journal of Applied Physics 114(8), 084314 (2013). doi:10.1063/1.4819799

27. Li, Y., Wan, Q., Jalii, M.B.A.: Magnetic field modulation of chiral tunneling in graphene heterojunctions. Journal of Physics D: Applied Physics 48(46), 465304 (2015). doi:10.1088/0022-3727/48/46/465304

28. Lafont, F., Ribeiro-Palau, R., Han, Z., Cresti, A., Delvallée, A., Cummings, A.W., Roche, S., Bouchiat, V., Ducourtieux, S., Schopfer, F., Poirier, W.: Anomalous dissipation mechanism and hall quantization limit in polycrystalline graphene grown by chemical vapor deposition. Physical Review B 90(11), 115422 (2014). doi:10.1103/PhysRevB.90.115422

29. Cummings, A.W., Cresti, A., Roche, S.: Quantum hall effect in polycrystalline graphene: The role of grain boundaries. Physical Review B 90(16), 2055 (2014). doi:10.1103/PhysRevB.90.161401

30. Dal Lago, V., Torres, L.E.F.F.: Line defects and quantum hall plateaus in graphene. Journal of physics. Condensed matter : an Institute of Physics journal 27(14), 145303 (2015). doi:10.1088/0953-8984/27/14/145303

31. Wang, S., Ren, C., Li, Y., Tian, H., Lu, W., Sun, M.: Spin and valley filter across line defect in silicene. Applied Physics Express 11(5), 053004 (2018). doi:10.7567/APEX.11.053004

32. Ren, C., Zhou, B., Sun, M., Wang, S., Li, Y., Tian, H., Lu, W.: Chiral filtration-induced spin/valley polarization in silicene line defects. Applied Physics Express 11(6), 063006 (2018). doi:10.7567/APEX.11.063006

33. Bahamon, D.A., Pereira, A.L.C., Schulz, P.A.: Third edge for a graphene nanoribbon: A tight-binding model calculation. Physical Review B 83(15), 155436 (2011). doi:10.1103/PhysRevB.83.155436

34. Cresti, A., Grosso, G., Parravicini, G.P.: Electronic states and magnetotransport in unipolar and bipolar graphene ribbons. Physical Review B 77(11), 620 (2008). doi:10.1103/PhysRevB.77.115408

35. Chen, J.-H., Autès, G., Alem, N., Gargiulo, F., Gautam, A., Linck, M., Kisielowski, C., Yazyev, O.V., Louie, S.G., Zettl, A.: Controlled growth of a line defect in graphene and implications for gate-tunable valley filtering. Physical Review B 89(12), 121407 (2014). doi:10.1103/PhysRevB.89.121407

\section{Figures}

Tables

Table 1 The ranges of $B$ (in units of $\mathrm{T}$ ) and $L$ (in units of $\sqrt{3} a / 2)$ to realize $T_{K}=1$ at $\alpha \approx \pi / 2$ for different energies.

\begin{tabular}{cccccc}
\hline Energy & range of $B$ & range of $L$ & Energy & range of $B$ & range of $L$ \\
\hline $0.001 t$ & {$[0.021,0.023]$} & {$[1692,1852]$} & $0.01 t$ & {$[2.26,2.34]$} & {$[164,184]$} \\
$0.003 t$ & {$[0.198,0.202]$} & {$[560,626]$} & $0.012 t$ & {$[3.29,3.40]$} & {$[136,152]$} \\
$0.005 t$ & {$[0.55,0.56]$} & {$[334,376]$} & $0.014 t$ & {$[4.53,4.67]$} & {$[116,130]$} \\
$0.008 t$ & {$[1.437,1.477]$} & {$[206,232]$} & $0.015 t$ & {$[5.23,5.40]$} & {$[108,120]$} \\
\hline
\end{tabular}


Figure 1 (Color online) (a) The graphene sheet with a line defect along the $y$-axis. The blue(red) line indicates the electron trajectory of the $K^{\prime}(K)$ valley in the magnetic field $B$ which is applied adjacent to the line defect. $L(L=4)$ represents the width of the perpendicular magnetic field $B$ region in units of $\sqrt{3} a / 2$, where $a(a=0.246 \mathrm{~nm})$ is the graphene lattice constant. (b) Schematic diagram illustrating the relationship between the peak transmission and the incident angle. $\Delta k$ is the shifting of the Dirac points along the $k_{y}$ axis, and the thick and thin arrows indicate that the peak transmission is maintained or weakened across the scattering region. (c)The simplified lattice model of the infinite graphene with a line defect. $\theta=k_{y} a$ is from the Fourier transformation along the $y$ axis. The dashed box represents the supercell and the parameters $t, \tau_{1}$ and $\tau_{2}$ denote various nearest-neighbor hopping energies in the tight-binding model.

Figure 2 (Color online) The transmission coefficients $T$ in $K(\mathrm{a})$ and $K^{\prime}(\mathrm{b})$ valleys are mapped as a function of the incident angle $\alpha$ for different magnetic field.

Figure 3 (Color online) Transmission coefficients $T_{K}(\mathrm{a})$ and $T_{K^{\prime}}$ (b) as a function of $\alpha$ (x-axis) and $B$-field ( $y$-axis). The blue line in (a) represents the relation between $\alpha$ and $B$ at which the peak transmission $T_{K}=1$ occurs according to Eq. (6).

Figure 4 (Color online) The critical magnetic field $B_{c}$ versus the corresponding width of the scattering region $L$ (in units of $\sqrt{3} a / 2$ ) in which $T_{K}=1$ occurs as $\alpha=\pi / 2$. The energy is $E=0.01 t$ in (a) and $E=0.005 t$ in (b). The transmission curve in (b) corresponds to the red circle in the $L-B_{c}$ panel.

Figure 5 (Color online) The transmission coefficients $T$ in $K$ (a) and $K^{\prime}$ (b) valleys as a function of the incident angle $\alpha$ for different on-site energys $E_{L}$ in the left electrode. Other parameters are $B=2.31 \mathrm{~T}, L=174$ and the electron energy besides the left electrode is $0.01 t$.

Figure 6 (Color online) The transmission coefficients $T$ in $K$ (a) and $K^{\prime}$ (b) valleys as a function of the incident angle $\alpha$ for different on-site potential strengths $W$. The on-site energys $E_{L}$ in the left electrode is $0.2 t$ and other parameters are the same as that in Fig .5. 


\section{Figures}

(a)

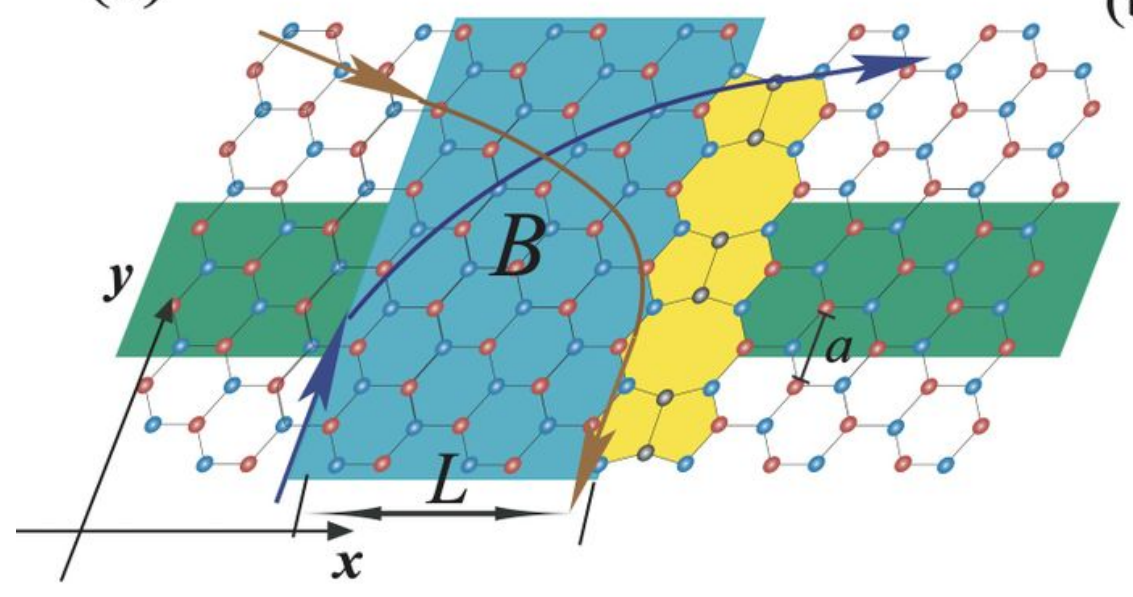

(c)

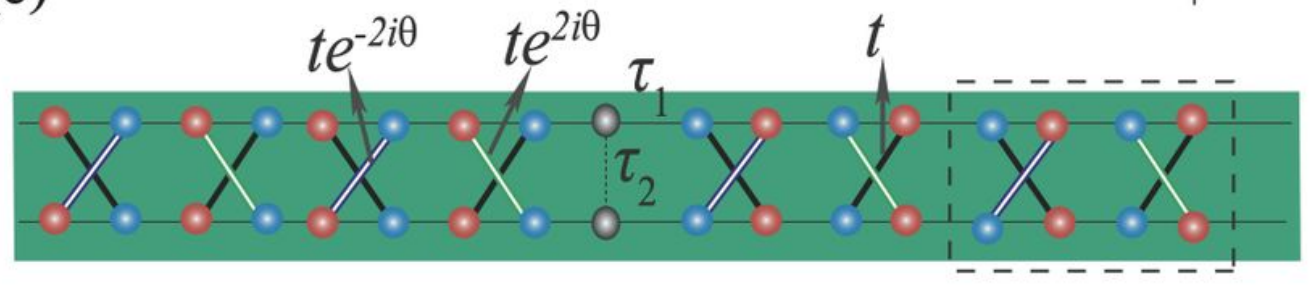

(b)

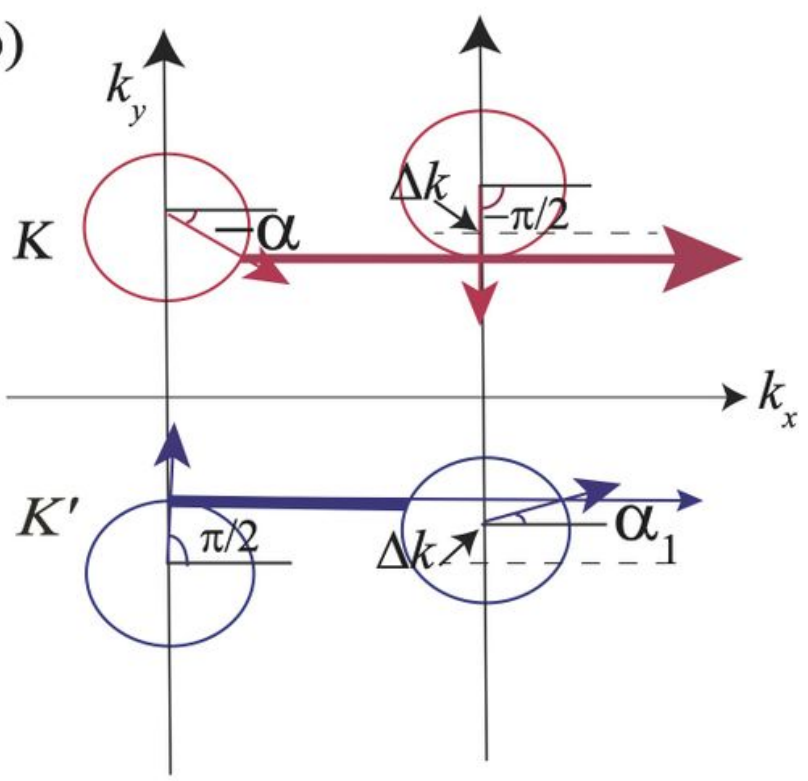

\section{Figure 1}

(Color online) (a) The graphene sheet with a line defect along the $y$-axis. The blue(red) line indicates the electron trajectory of the $K^{\prime}(K)$ valley in the magnetic field $B$ which is applied adjacent to the line defect. $L(L=4)$ represents the width of the perpendicular magnetic field $B$ region in units of $\sqrt{ } 3 a / 2$, where $a(a=$ $0.246 \mathrm{~nm}$ ) is the graphene lattice constant. (b) Schematic diagram illustrating the relationship between the peak transmission and the incident angle. $\Delta \mathrm{k}$ is the shifting of the Dirac points along the ky axis, and the thick and thin arrows indicate that the peak transmission is maintained or weakened across the scattering region. (c)The simplified lattice model of the infinite graphene with a line defect. $\theta=$ kya is from the Fourier transformation along the y axis. The dashed box represents the supercell and the parameters $t, \tau 1$ and $\tau 2$ denote various nearest-neighbor hopping energies in the tight-binding model. 


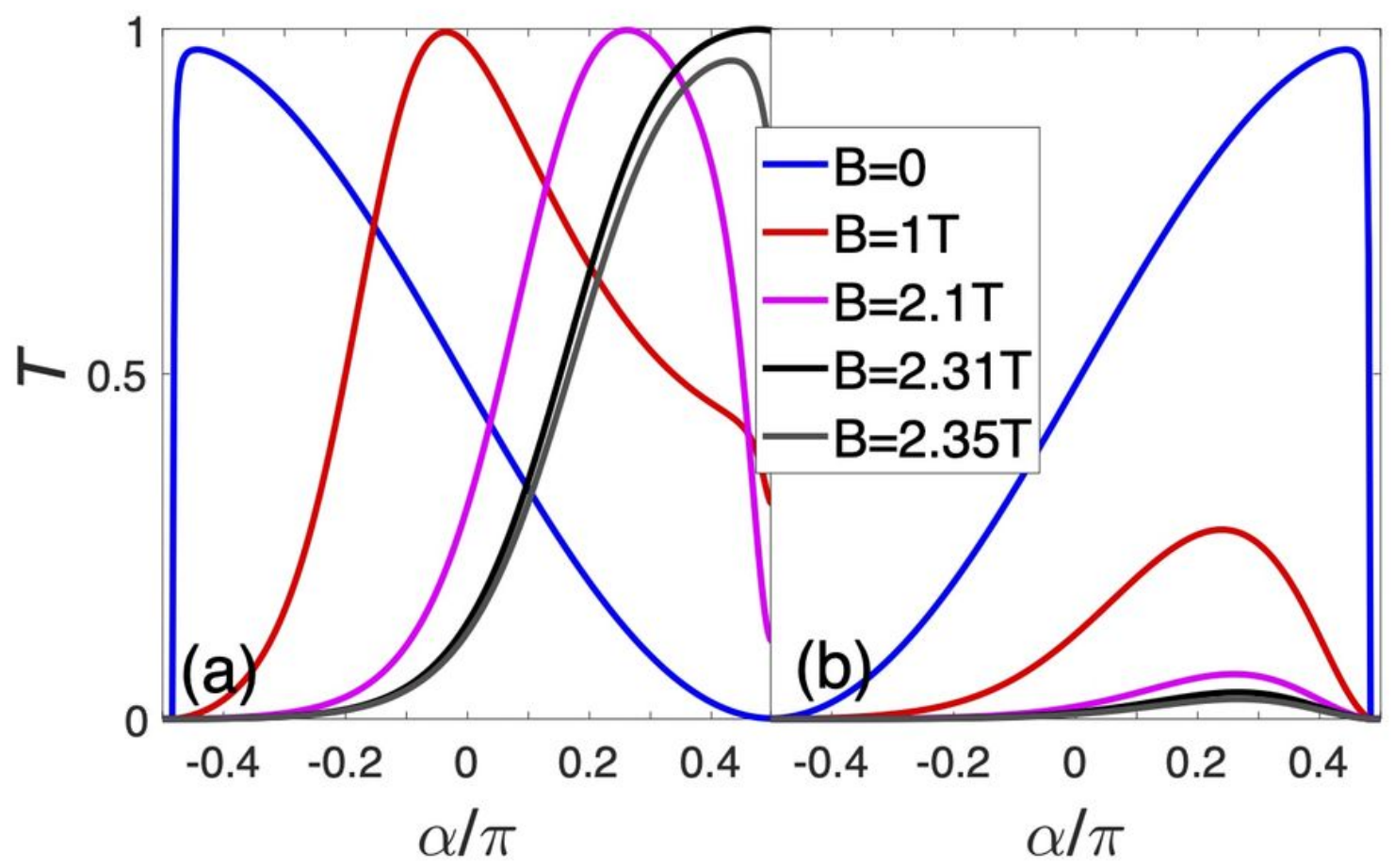

Figure 3

(Color online) The transmission coefficients $\mathrm{T}$ in $\mathrm{K}(\mathrm{a})$ and $\mathrm{K}^{\prime}(\mathrm{b})$ valleys are mapped as a function of the incident angle a for different magnetic field.

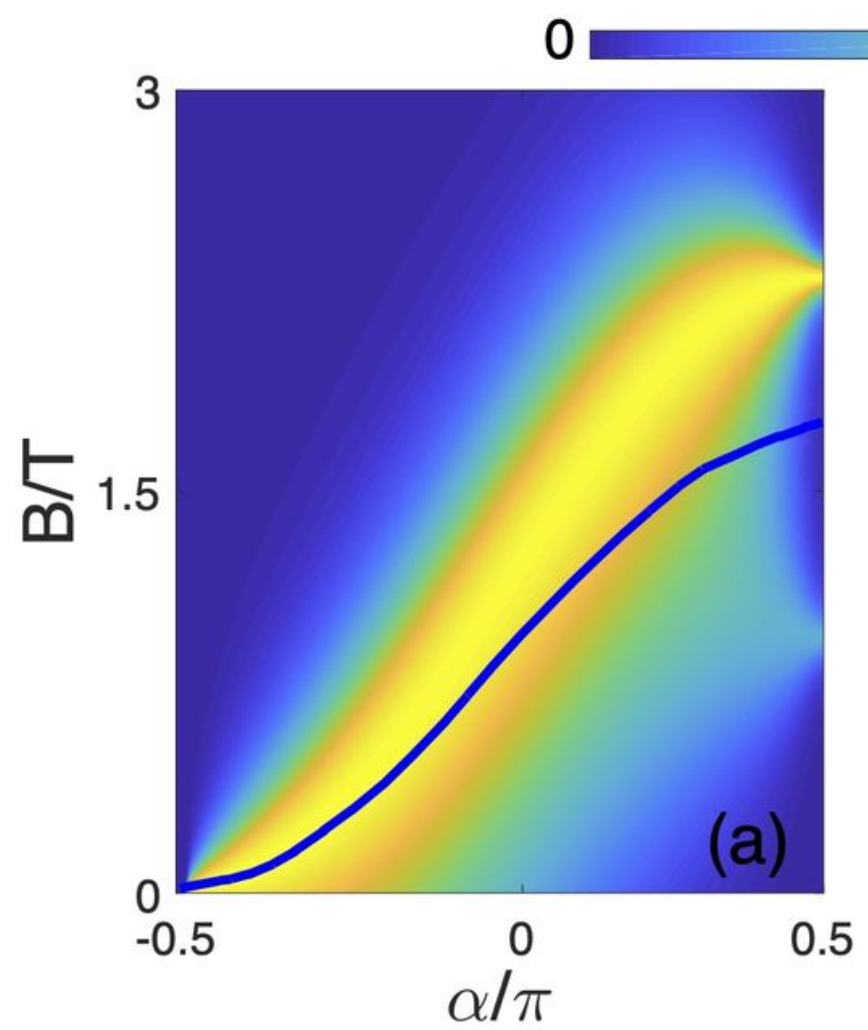

\section{1}

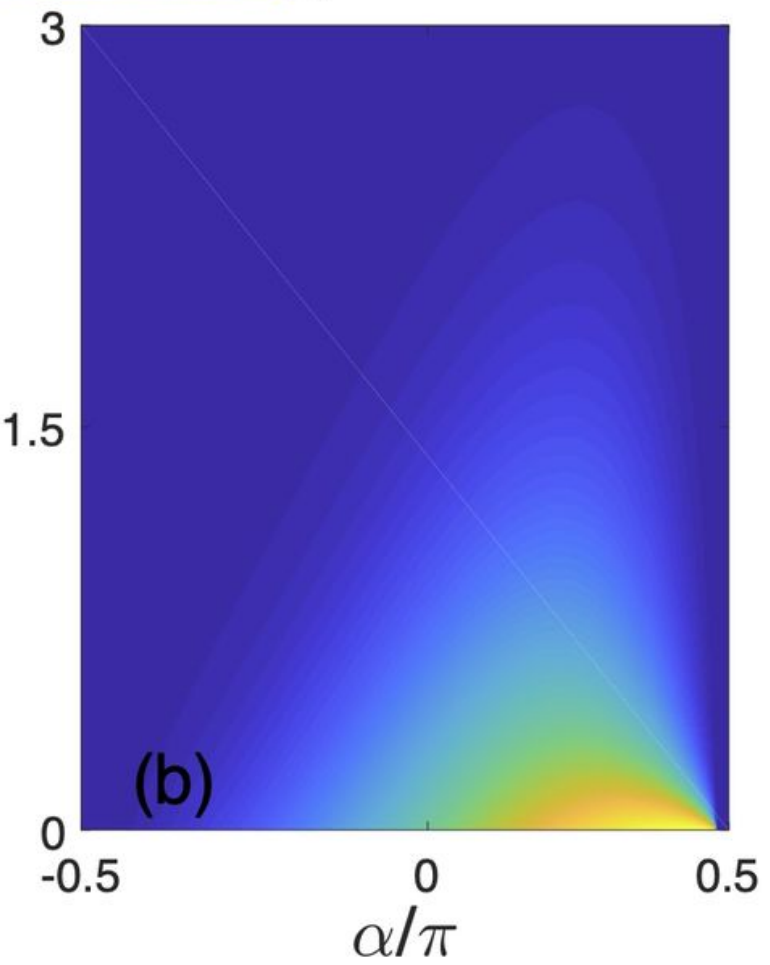

Figure 5 
(Color online) Transmission coefficients TK (a) and TK' (b) as a function of a (x-axis) and B-field (y-axis). The blue line in (a) represents the relation between $a$ and $B$ at which the peak transmission TK $=1$ occurs according to Eq. (6).

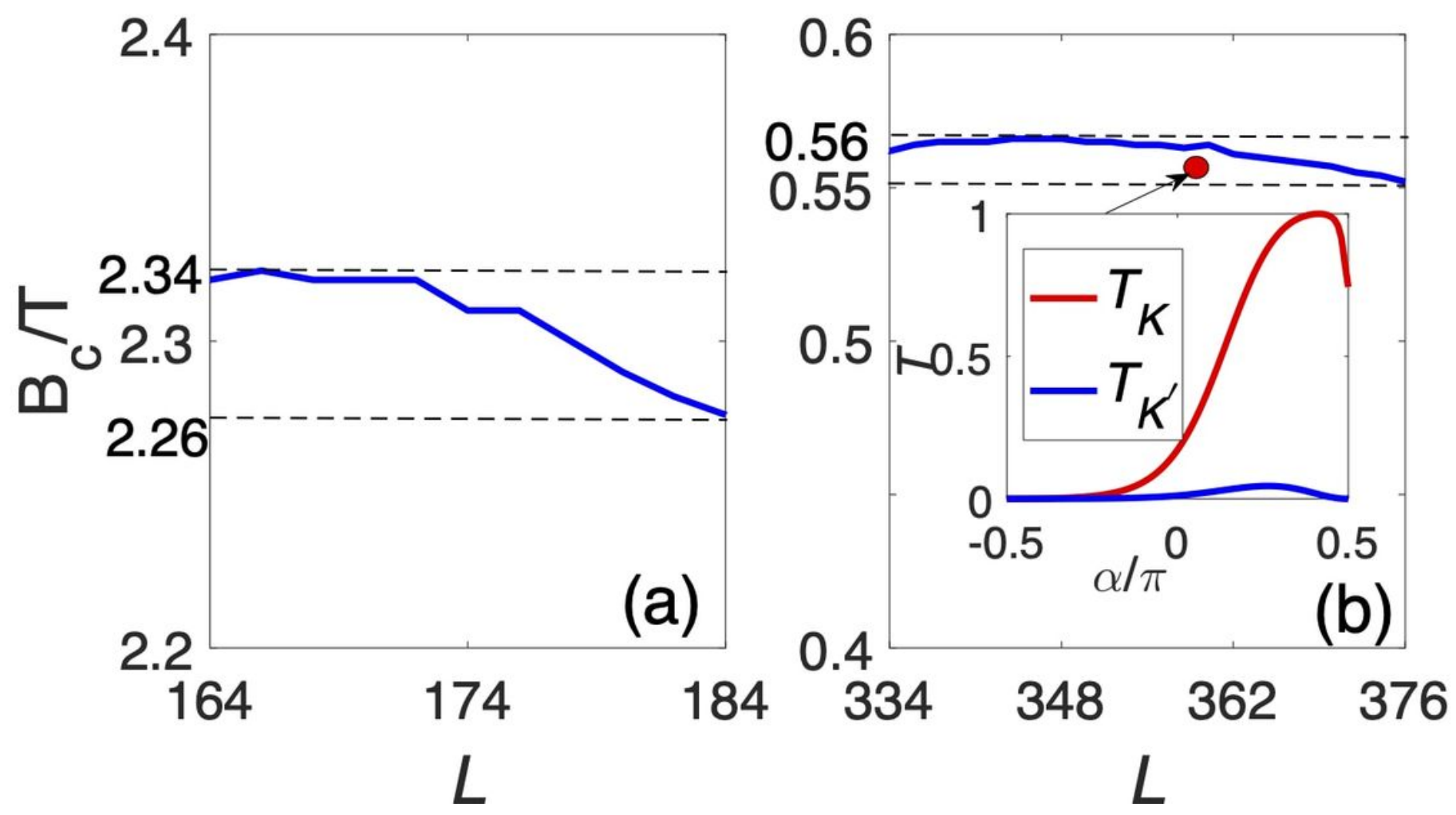

Figure 7

(Color online) The critical magnetic field $\mathrm{Bc}$ versus the corresponding width of the scattering region $\mathrm{L}$ (in units of $\sqrt{ } 3 a / 2$ ) in which $T K=1$ occurs as $a=\pi / 2$. The energy is $E=0.01 t$ in (a) and $E=0.005 t$ in (b). The transmission curve in (b) corresponds to the red circle in the $L-B c$ panel.
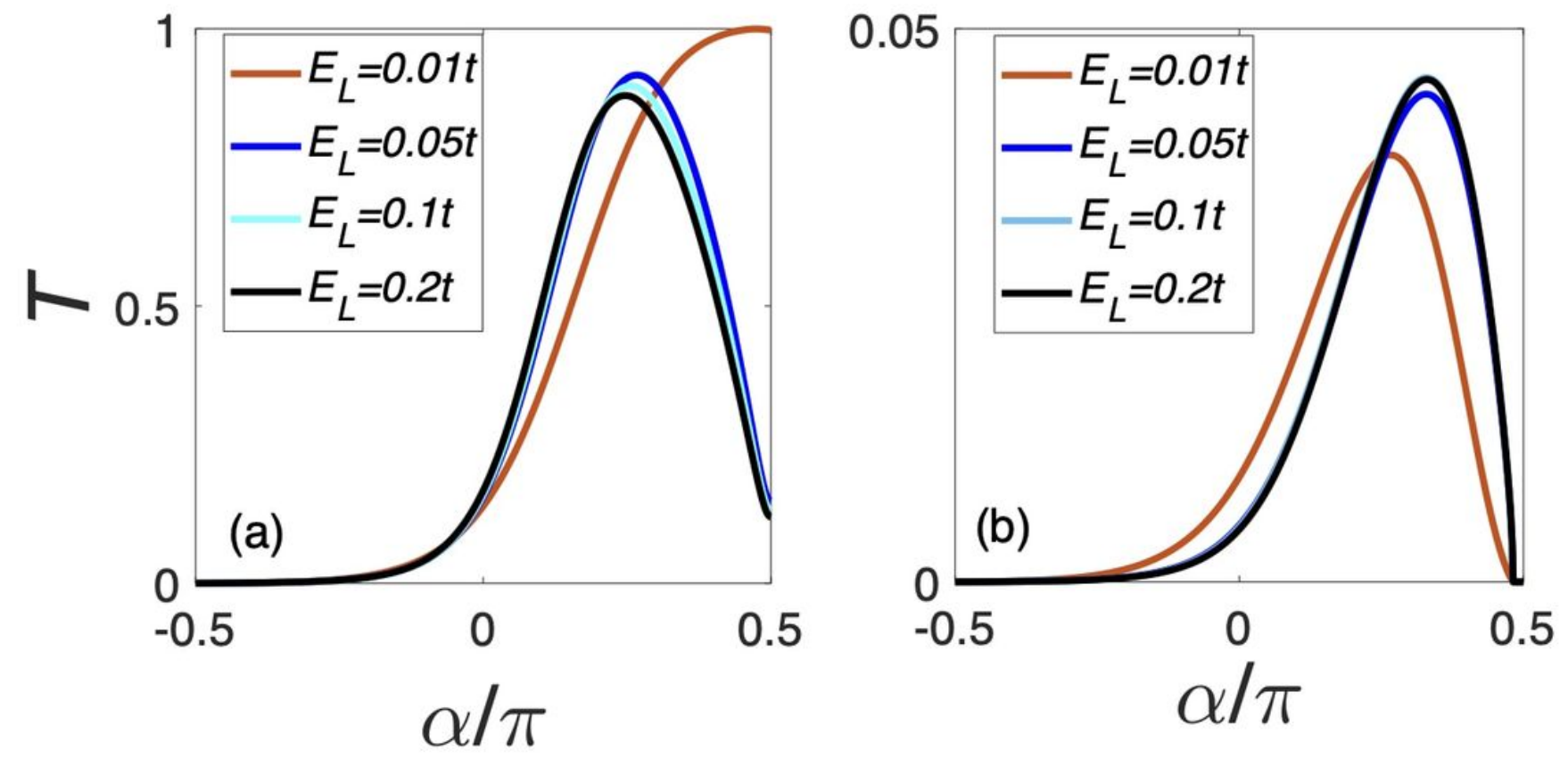
Figure 9

(Color online) The transmission coefficients $\mathrm{T}$ in $\mathrm{K}(\mathrm{a})$ and $\mathrm{K}^{\prime}(\mathrm{b})$ valleys as a function of the incident angle a for different on-site energys $E L$ in the left electrode. Other parameters are $B=2.31 T, L=174$ and the electron energy besides the left electrode is $0.01 \mathrm{t}$.
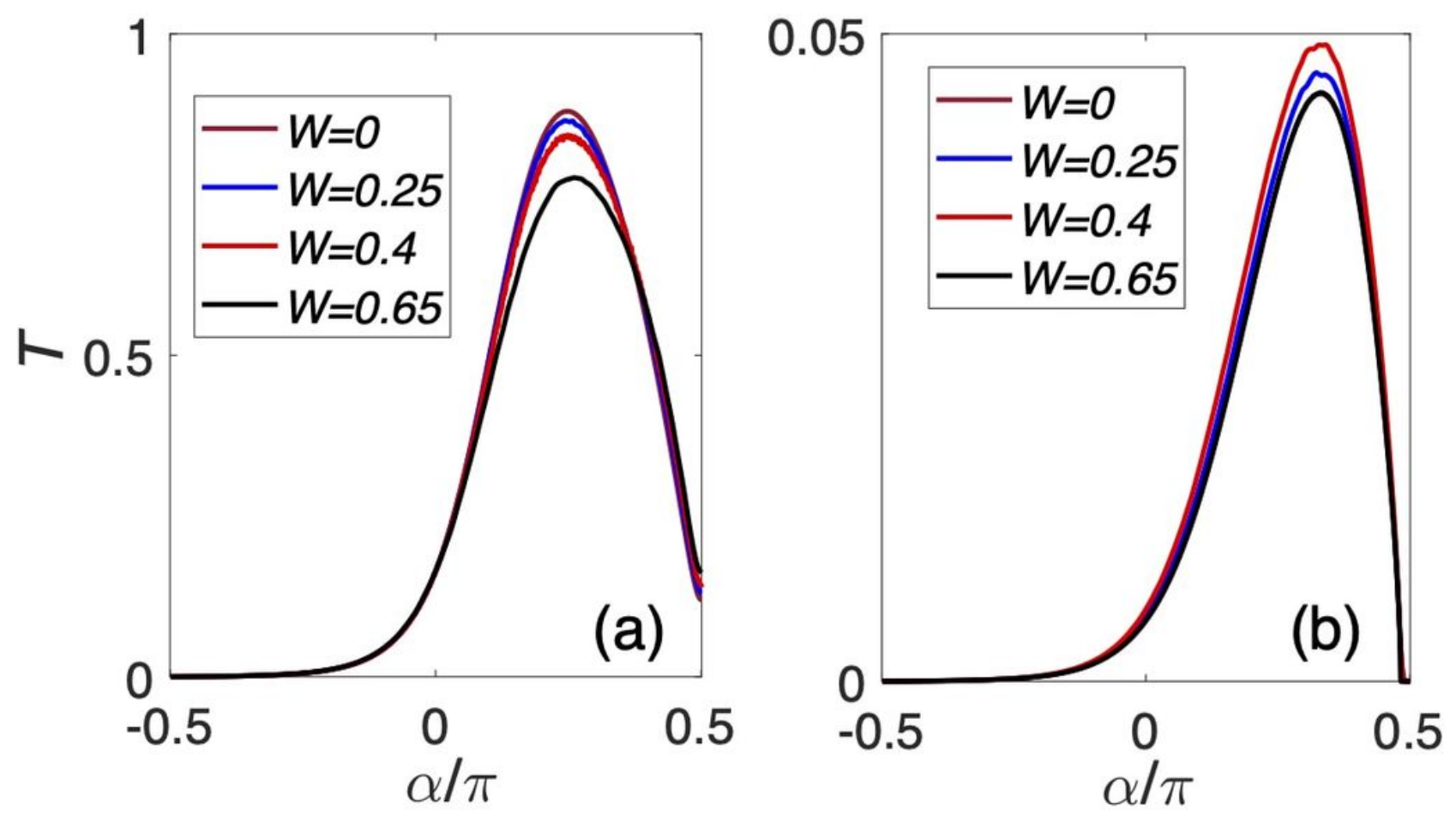

Figure 11

(Color online) The transmission coefficients $\mathrm{T}$ in $\mathrm{K}(\mathrm{a})$ and $\mathrm{K}^{\prime}(\mathrm{b})$ valleys as a function of the incident angle a for different on-site potential strengths $W$. The on-site energys $E L$ in the left electrode is $0.2 t$ and other parameters are the same as that in Fig .5.

\section{Supplementary Files}

This is a list of supplementary files associated with this preprint. Click to download.

- tian3.bib

- bmcarticle.tex

- tian3.bib

- bmcarticle.tex 